\title{
Las representaciones numéricas básicas en los deficientes auditivos profundos
}

\author{
JOSÉ MIGUEL RODRÍGUEZ SANTOS, JAVIER GARCÍA ORZA \\ Y MARINA CALLEJA REINA \\ Universidad de Málaga
}

\section{INTRODUCCIÓN}

LOS NÚMEROS SON UNA adquisición cultural muy reciente en la evolución de la especie humana. Y aún más reciente es la aparición de las palabras numéricas y de los dígitos, los cuales proceden de una habilidad específicamente humana para crear y manipular mentalmente símbolos complejos. Sin embargo, el sentido numérico es bastante más viejo. La sensibilidad a las propiedades numéricas del entorno está presente no sólo en el ser humano sino incluso en muchas otras especies no humanas. El hecho de que podamos sostener que muchas especies no humanas poseen este sentido numérico o que pueden incluir nociones de tipo numérico en su comportamiento, nos sugiere que la representación interna de los números tanto en los animales no humanos como en los seres humanos tiene que ser bastante abstracta (Gallistel y Gelman, 1992).

En la actualidad, existe un consenso generalizado de que existe un "sentido numérico" preverbal básico, una especie de representación mental aproximada de las magnitudes. Esta habilidad para discriminar conjuntos basándose en la cantidad de los elementos que los componen -la numerosidad o magnitud de los mismos- también la podemos encontrar en los bebés humanos preverbales. Utilizando métodos clásicos de habituación y de tiempo de recuperación de la mirada -un paradigma experimental ampliamente utilizado en estudios con bebés e incluyendo tanto los bebés recién nacidos como los bebés un poquito mayores aunque todavía preverbales- se ha podido comprobar que estos bebés son capaces de discriminar entre conjuntos de objetos visuales, entre tonos de sonidos o entre palabras que difieren en el número de sílabas, basándose únicamente en su cálculo de la magnitud, en la numerosidad presente en el conjunto (Xu y Spelke, 2000). Podemos, por tanto, mostrar evidencias de un emparejamiento entre conjuntos de elementos que se basa únicamente en 
las magnitudes internas y que, además, pueden ser de naturaleza transmodal. Diversas líneas de investigación tienden también a conceder credibilidad a la hipótesis de que el sentido numérico preverbal es el que proporciona el fundamento para la matemática formal posterior. La argumentación se basa en dos tipos de evidencias: En primer lugar, la evidencia neurológica que muestra que muchas de las tareas que implican explícitamente cantidades formuladas de manera simbólica activan de manera automática las representaciones numéricas no simbólicas (Dehaene, Dupoux y Mehler, 1990). En segundo lugar, los adultos humanos son capaces de realizar un cálculo aritmético aproximado de manera muy automática mediante dígitos arábigos, utilizando aparentemente para ello un circuito cerebral distinto del que está implicado en el cálculo numérico exacto (Piazza y Dehaene, 2005, para una revisión). A pesar de estas argumentaciones, esta última evidencia se ha mantenido durante algún tiempo como una cuestión no bien resuelta. No quedaba del todo claro si las representaciones no simbólicas de las magnitudes podían servir, realmente, como fundamento de la matemática formal posterior. Para que esta hipótesis fuese cierta, debería ser posible realizar operaciones aritméticas con representaciones aproximadas de las magnitudes sin tener que utilizar procesos verbales o simbólicos. Esta cuestión ha sido afrontada recientemente por Barth, La Mont, Lipton, Dehaene, Kanwisher y Spelke (2006) quienes investigaron esta cuestión de una forma directa preguntándose si los adultos oyentes y los niños preescolares oyentes podían realizar operaciones de cálculo aritmético simple mediante magnitudes no simbólicas. Sus resultados pusieron claramente de manifiesto que tanto los adultos como los niños preescolares, éstos últimos sin entrenamiento alguno en tareas de cálculo aritmético, realizaban de manera exitosa cálculos aritméticos en conjuntos compuestos por grandes cantidades de elementos (normalmente conjuntos de puntos de gran tamaño). El éxito en estas tareas no dependía de que hubiera cantidades no numéricas continuas -por ejemplo superficies-, tampoco dependía de que existiera una información cuantitativa específica de modalidad o de la adopción de un conocimiento alternativo de naturaleza no aritmética que permitiera resolver el problema. Así, pues, estos autores concluyeron que las representaciones numéricas de estas cantidades poseían varias propiedades: tenían una naturaleza abstracta, tenían también una naturaleza computacional -se podía operar sobre ellas- y podían proporcionar el fundamento para la matemática formal necesaria para gestionar nuestra vida cotidiana. Las propiedades de estas habilidades numéricas se manifiestan, en grados diversos, en muchas ocupaciones cotidianas: manejar cambio de moneda en un supermercado, calcular el descuento en una compra o elaborar un pequeño presupuesto, por nombrar algunos ejemplos (McCloskey, 2007). Sin embargo, a pesar de la omnipresencia de las realidades numéricas y a pesar del nivel básico de estas habilidades, no todo el mundo encuentra fáciles los 
conceptos numéricos. Los datos demográficos nos indican que entre el 5\% y el $10 \%$ de los niños y adolescentes oyentes experimentan algún problema en el aprendizaje de algún área matemática (Shalev, 2007, para una revisión).

Estos hallazgos sobre el procesamiento numérico fueron muy prometedores y nos permitieron mejorar nuestra comprensión del desarrollo de las habilidades numéricas de los niños, y en particular en los niños sordos al aplicar los hallazgos anteriores. A partir de los estudios iniciales de los años 90, en la última década se ha desarrollado un conjunto de estudios que intentan explicar la naturaleza de las representaciones numéricas en los niños sordos.

\section{LAS DIFICULTADES DE LAS PERSONAS SORDAS CON LAS REPRESENTACIONES NUMÉRICAS}

La evidencia procedente de diversas líneas de investigación, desarrolladas entre los años 1980 y 2000, muestra de manera consistente que los niños sordos presentan un desfase en relación a los niños oyentes de entre 2 y 3,5 años en los tests de rendimiento matemático. El estudio más relevante fue el realizado por Wood et al. (1984). Estos autores encontraron que la edad matemática de los niños oyentes al término de la escolaridad era de alrededor de 15,5 años frente a la edad matemática de los niños sordos que era de 12,3 años. Un dato importante era que, a diferencia de los datos que conocemos sobre el rendimiento lector, se ha encontrado que el $15 \%$ de los niños sordos tenían edades matemáticas equivalentes a su edad cronológica o incluso por encima de la misma, mientras que la proporción de niños sordos que presentan edades lectoras por encima de su edad o incluso equivalente es prácticamente marginal.

No obstante lo anterior, es preciso remarcar que muchos de los trabajos de investigación publicados se refieren al rendimiento matemático como una parte más dentro de estudios más amplios de rendimiento educativo general donde, muy frecuentemente, el foco central de interés era la lectura. Este énfasis en la lectura ha resultado útil, en cierta medida, dado que las habilidades de lenguaje se consideran necesarias para el acceso a la información numérica matemática. Además, el rendimiento en matemáticas tiende a covariar con el rendimiento en lectura. Sin embargo, hasta no hace mucho escaseaban los estudios que nos proporcionan una información específica de las habilidades matemáticas de los niños sordos en sí mismas o que nos ilustraran sobre otros factores tales como la experiencia de aprendizaje o el impacto debido a las dificultades en el acceso al currículum educativo (ver Powers et al. 1998 para una revisión).

A partir del conocimiento que tenemos sobre el desarrollo de la cognición numérica en las personas oyentes podemos intentar comprender el déficit frecuentemente observado en el rendimiento aritmético y matemático de los 
niños y adultos sordos (Lang, 2003; Traxler, 2000). Cuando aplicamos tests estandarizados de rendimiento académico, los retrasos en el rendimiento matemático aparecen aproximadamente a partir de los ocho años de edad (Traxler, 2000) y permanecen relativamente constantes. Los trabajos que han estudiado los aspectos específicos de la comprensión matemática han mostrado que los estudiantes sordos presentan retrasos en los conceptos de medida y de número (Austin, 1975), en los conceptos de fracciones o quebrados (Titus, 1995). También presentan problemas de comparación aritmética (Kelly et al., 2003).

\section{II.1. EXPLICACIONES GENERALES DEL DÉFICIT: EXPERIENCIA Y LENGUAJE}

Sin duda, las primeras experiencias educativas y de aprendizaje de los niños sordos tienen que desempeñar un papel importante en la forma en que se desarrollan las habilidades numéricas (Marschark, Lang y Albertini, 2002), aunque son pocos los estudios que han considerado específicamente la forma en que las diferencias en procesamiento general mostradas por las personas sordas pueden influir en el aprendizaje y representación del conocimiento numérico (Schick, 2005). Como ya hemos mencionado anteriormente, la mayor parte de los estudios que han analizado las habilidades matemáticas de las poblaciones sordas ha observado únicamente el rendimiento matemático como parte de estudios más amplios de rendimiento educativo en general, en el que frecuentemente el centro de atención era la capacidad lectora (Swanwick et $a l ., 2005)$. Las habilidades lingüísticas se consideran importantes en tanto en cuanto permiten el acceso a la formación matemática y en los estudios tanto en sordos como en oyentes se observa frecuentemente una fuerte asociación entre las habilidades del lenguaje y las habilidades matemáticas (Hyde, Zevenbergen y Power, 2003). Algunos investigadores incluso han llegado a argumentar que el lenguaje es la barrera clave para el éxito matemático (Swanwick, Oddy y Roper, 2005). Y es cierto que esta afirmación es especialmente pertinente cuando se refiere a los problemas matemáticos que incluyen enunciados, puesto que es entonces cuando la comprensión del lenguaje y su traducción a los términos operativos del problema resulta decisiva. Otros autores argumentan que esta percepción ha conducido a descuidar el proceso completo de procesamiento que tiene lugar en la resolución de problemas (Kelly, Lang y Pagliaro, 2003) porque es evidente que no solamente es el lenguaje la variable que influye en la comprensión numérica y en el desarrollo de las habilidades matemáticas.

Sin embargo, a pesar del innegable impacto que tienen las habilidades lingüísticas en el desarrollo posterior de la matemática formal, muchas tareas aritméticas no necesitan recurrir al lenguaje para ser ejecutadas y también podemos observar que hay una clara evidencia neuropsicológica que muestra que aquellas habilidades que tienen un fuerte componente verbal (tal como el 
cálculo exacto) y aquellas habilidades numéricas que no tienen una base verbal (tales como la estimación o la comparación de magnitudes) activan áreas cerebrales diferentes tal como han puesto de relieve los estudios con neuroimagen (Dehaene, Dupoux y Mehler, 1990) . Por otro lado, la habilidad de los bebés para responder a las propiedades numéricas es también una prueba del hecho de que algunos aspectos del procesamiento numérico no se apoyan en las habilidades verbales o lingüísticas (Butterworth, 2005).

\section{LAS HABILIDADES NUMÉRICAS BÁSICAS EN LOS DEFICIENTES AUDITIVOS PROFUNDOS}

Para plantearnos todas estas cuestiones anteriores procederemos a revisar los estudios sobre cognición numérica que se han realizado con personas oyentes e intentaremos mostrar cómo las estrategias de procesamiento de la información de las personas sordas pueden estar influyendo en el aprendizaje, la representación y la recuperación del conocimiento matemático. La información procedente de un amplio conjunto de tareas que evalúan el reconocimiento de la cantidad, la representación de magnitudes o la recuperación de magnitudes nos va a servir como punto de partida para identificar si estas habilidades numéricas básicas explicarían las dificultades en el desarrollo de las habilidades matemáticas o, tan importante como lo primero, si pueden ser descartadas como factores que contribuyen a estas dificultades matemáticas.

\section{III.1. LA RECUPERACIÓN DE LA INFORMACIÓN SOBRE LAS MAGNITUDES}

La teoría del triple código de Dehaene asume que cuando los numerales arábigos o verbales son identificados, la información numérica se transfiere automáticamente a una representación de magnitudes de tipo analógico y no verbal. Esta representación incluye información semántica como puede ser la referente a la cantidad relativa y contribuye al rendimiento matemático (Dehaene, 1992). Una deficitaria representación no verbal de las magnitudes - p. e. en la estimación aproximada- puede interferir con el desarrollo de las habilidades de cálculo exacto (Ansari y Karmiloff-Smith, 2002). Los investigadores han sugerido que los trastornos del cálculo, la discalculia, implican un déficit en la representación de cantidades o de su acceso mediante las representaciones simbólicas (Berch, 2005; Butterworth, 2005). Una representación mental numérica poco clara o poco precisa se traducirá en una mayor dificultad para establecer lazos robustos entre los símbolos numéricos, tales como los numerales arábigos, y su información semántica asociada. Esta deficitaria representación numérica se traducirá en dificultades en tareas tales como la comparación de 
magnitudes o la de estimación de magnitudes y también se traducirá en una mayor variabilidad entre los límites de las diferentes magnitudes a lo largo de una línea mental numérica. Un ejemplo de este dato es el estudio de Rubinstein y Henik (2005) quienes encontraron que los adultos con discalculia evolutiva presentaban problemas para la activación automática de las magnitudes usando dígitos, mostrando, en cambio, una menor interferencia en las tareas de comparación de tamaño físico cuando el tamaño físico de los dígitos era incongruente con su magnitud -Stroop numérico. Además, Jordan, Hanich y Kaplan (2003) sostuvieron que una representación espacial débil o inadecuada de las magnitudes numéricas, en concreto las dificultades para manipular la representación visual en una línea mental numérica, eran particularmente importantes o influyentes durante la adquisición temprana de las habilidades de cálculo y que podían subyacer a los déficits de recuperación aritmética observadas en muchas poblaciones con dificultades matemáticas.

\section{III.2. EL AUTOMATISMO EN LA RECUPERACIÓN DE LA INFORMACIÓN ACERCA DE} LA MAGNITUD

El método más comúnmente utilizado para estudiar la forma de recuperación de la información numérica o de magnitudes ha sido el paradigma Stroop (ver MacLeod, 1991 para una revisión). Este procedimiento experimental evalúa la interferencia procedente de un aspecto irrelevante del estímulo en relación a otro que se considera relevante. Un ejemplo de una tarea numérica de tipo Stroop implica pedir a los participantes que indiquen qué número es físicamente mayor cuando se les presentan dos estímulos que son congruentes (tamaño físico y magnitud coinciden, p. e. 1 y 3 ), ${ }^{1}$ incongruentes (cuando el tamaño físico y la magnitud no coinciden: 1 y 3$)^{2}$ o neutrales (cuando los estímulos coinciden en la magnitud pero no en el tamaño: 3 y 3 ). ${ }^{3}$ Los resultados muestran que en la condición de incongruente, es decir, cuando no coinciden la magnitud indicada por el número con su tamaño físico, dicha magnitud interfiere con los juicios de tamaño físico, mostrando el denominado efecto de congruencia; la consecuencia es que se ralentizan los tiempos de respuesta en las condiciones de incongruente comparados con los tiempos obtenidos en las condiciones neutrales y, por otro lado, se aceleran los tiempos de respuesta en las situaciones de congruente comparados con los tiempos obtenidos en las condiciones neutrales. Las diferencias en los tiempos de respuesta nos muestran el tamaño del efecto de congruencia (Rubinstein y Henik, 2005).

1 Obsérvese que el tamaño físico es el elemento relevante en este caso.

2 En este caso, el número de menor magnitud (el 1) es el mayor de tamaño físico.

3 En este caso, las magnitudes son iguales pero los tamaños físicos no. 
Los estudios realizados con niños oyentes muestran que estos efectos de congruencia no están presentes en el inicio de la escolarización de los niños pero están presentes posteriormente en los niños de tercer grado (nueve años aproximadamente) y mayores, lo que sugiere un proceso gradual de automatización en el procesamiento a medida que las habilidades numéricas avanzan (Girelli et al., 2000). Muchos investigadores han argumentado que las diferencias entre sordos y oyentes en la cantidad de exposición incidental a las nociones numéricas - experiencia directa de las nociones numéricas- $y$, posteriormente, a la instrucción matemática, podrían explicar la diferencia o desfase tanto en la capacidad para el procesamiento numérico como en la capacidad para el cálculo que se observa entre las personas sordas y las personas oyentes (Epstein et al., 1994; Gregory, 1998). Si los niños sordos han tenido muchas menos oportunidades para practicar las habilidades numéricas, entonces es normal que exista un retraso en la automatización de las habilidades numéricas y esto llevaría a una menor presencia o incluso a la total ausencia de los denominados efectos de congruencia.

Además de esta explicación otra posible es la explicación lingüística. Bull et al. (2008) realizaron un estudio en el que la diferencia entre los sujetos sordos de su estudio radicaba en la modalidad comunicativa utilizada. De los niños sordos estudiados, algunos utilizaban el lenguaje de signos $\left(\mathrm{BSL}^{4}\right)$. En concreto, fueron tres niños los que lo utilizaban como su forma principal de comunicación en la escuela y únicamente uno de estos niños mostró efectos de interferencia. Todos los demás niños utilizaban el lenguaje oral y doce de los quince niños mostraron los efectos de interferencia.

Aunque es difícil extraer conclusiones sólidas basándose en tales informaciones a partir de una muestra tan reducida, podríamos centrarnos con mayor detalle en las posibles diferencias en función del uso de una u otra modalidad comunicativa. Esto es especialmente relevante si se tiene en cuenta que la modalidad comunicativa tiene un impacto considerable en las oportunidades de aprendizaje incidental temprano de las habilidades numéricas. Bandurski y Galkowski (2004)) encontraron diferencias claras entre los niños sordos hijos de padres sordos comparados con los niños sordos hijos de padres oyentes en tareas de procesamiento relacional, en el que se requería el uso de símbolos tanto matemáticos como lingüísticos. No hubo diferencia entre estos grupos en una versión de la tarea de naturaleza figural-geométrica, lo que sugería que las diferencias entre los grupos no estaban relacionadas, en general, con el procesamiento relacional en sí mismo, sino que eran debidas, más bien, a la ausencia de oportunidades de los niños sordos hijos de padres sordos para tener una exposición incidental a los símbolos matemáticos y lingüísticos. 
Esta variable, el tipo de modalidad comunicativa, fue también estudiada en un trabajo previo de Bull, Blatto-Vallee y Fabich (2006), usando igualmente el paradigma Stroop y los efectos de congruencia. En este estudio, todos los sujetos sordos utilizaban el lenguaje americano de signos (American Sign Language o ASL) como medio principal de comunicación. En este caso, además de utilizar numerales arábigos se incluyeron los numerales en formato ASL. En el caso de los símbolos arábigos, tanto los sordos como los oyentes adultos mostraron el efecto de congruencia tanto cuando hacían juicios de magnitud como cuando hacían juicios de tamaño físico, manteniéndose un tamaño del efecto de congruencia en magnitud similar para los sordos y para los oyentes. Cuando se realizó el mismo tipo de análisis sobre los símbolos numerales en ASL no se halló un efecto de congruencia en función del status auditivo de los sujetos (sordos u oyentes).

\section{III.3. LA FORMA DE LA REPRESENTACIÓN DE LAS MAGNITUDES: LA LÍNEA MENTAL}

El paradigma Stroop utilizado para estudiar el acceso automático a la información de magnitud ha permitido también el análisis de otros aspectos de la representación. Diversos estudios han informado que la información de magnitudes se representa conceptualmente bajo la forma de una línea mental visual, una línea visual que va de izquierda a derecha, estando representadas las cantidades más bajas en el lado izquierdo de la línea y las cantidades más altas en la parte derecha de la línea. Es decir, existe una asociación entre los códigos numéricos y los códigos espaciales. Esta asociación se conoce con el nombre de efecto SNARC (Spatial Number Association Response Code: asociación numérica espacial de los códigos de respuesta). Este efecto fue puesto de manifiesto por Dehaene et al. (1993). Este efecto ha sido considerado como una indicación de que la información de magnitud está siendo activada y que influye en otros juicios que no tienen nada que ver con la magnitud. Y esto ocurre incluso cuando la magnitud es irrelevante para la tarea. Así, pues, el efecto SNARC, aunque tiene el cometido primario de informarnos acerca de la forma de la representación de la información numérica en la memoria, no solamente nos proporciona información acerca del formato espacial de dicha representación, sino que también nos informa del grado de automatización que presenta.

Una evidencia adicional para esta forma de representación de magnitud y de traducción automática de los números escritos u oídos en magnitudes analógicas procede de los estudios de los efectos de distancia en las comparaciones numéricas (Dehaene et al., 1998). Cuando los números están cerca unos de otros en la línea mental (por ejemplo el 4 y el 5), el tiempo empleado en decidir cuál es el número mayor será más largo comparado con el tiempo 
obtenido en aquellos pares de números que estén más distantes en la línea numérica (como, por ejemplo, el 1 y el 5). Esto es debido a la imprecisión de las representaciones de magnitud, lo que produce grados de solapamiento o de borrosidad entre los límites de las magnitudes, haciendo particularmente difícil comparar magnitudes que estén muy cerca unas de otras.

En un estudio acerca de la representación numérica (Bull et al., 2005) examinaron si el procesamiento intencional de la magnitud produciría el típico efecto SNARC y el típico efecto de distancia. Es decir, estos trabajos se preocuparon por saber si los adultos sordos mostraban el mismo tipo de representación espacial de la magnitud. A los participantes se les pidió que realizaran alguna tarea simple de juicio de magnitudes: se les presentaron pares de números del 1 al 4 y del 6 al 9 y en una condición se les pidió que respondieran con la mano izquierda si el número era inferior a cinco y con la mano derecha si el número era superior a cinco. En la segunda condición las correspondencias de respuesta se invirtieron. Si los sujetos sordos mantenían la representación lineal de izquierda a derecha, se esperaría que mostraran el típico efecto SNARC (respuestas más rápidas a los números más bajos cuando se asocia con la izquierda y a los números altos con la mano derecha). También se esperaría encontrar el efecto de distancia, esto es, unos mayores tiempos de respuesta a aquellos números que estaban mucho más cercanos los unos de los otros, como por ejemplo el 4 y el 6 comparados con los que mantenían una mayor distancia entre sí, por ejemplo el 1 y el 9). En consonancia con los hallazgos encontrados en numerosos estudios con sujetos oyentes, los adultos sordos mostraron el mismo patrón de resultados. Tanto los adultos sordos como los adultos oyentes mostraron el efecto de distancia, mostrando respuestas más lentas a las relaciones 4-6 (cuando el número de comparación era el 5) comparados con todos los demás números.

Así, pues, podemos concluir que los hallazgos comparativos entre adultos sordos y adultos oyentes en tareas diseñadas para examinar el grado de automatismo en el acceso a información de magnitud y el formato espacial de la representación nos han mostrado que no hay diferencias significativas. Ambos grupos muestran resultados que indican una línea espacial orientada de izquierda a derecha, tal como muestran los efectos de distancia y de SNARC. También muestran la activación automática de la información de magnitud, tal como se evidencia por los efectos de congruencia. Por tanto, es poco probable que las dificultades en el aprendizaje de las matemáticas procedan de un problema básico en la representación o en el acceso a la información de magnitud. Los datos encontrados en estudios con los niños sordos más bien sugieren la existencia de amplias diferencias individuales en el grado de automatismo en el acceso a la información acerca de las magnitudes. 


\section{III.4. LA REPRESENTACIÓN DE MAGNITUDES ALTAS Y BAJAS EN EL PROCESO DE ENUMERACIÓN}

La habilidad para enumerar se puede descomponer en dos procesos básicos en función de las magnitudes a las que se aplique. En aquellos conjuntos que contengan un número pequeño de ítems (hasta 4 , que es lo que normalmente se conoce como el rango de subitizing), los sujetos son capaces de captar rápidamente y con precisión tal cantidad, su 'numerosidad' (Peterson y Simon, 2000). Los tiempos de respuesta sugieren que todos los ítems son captados simultáneamente y que hay pocas diferencias entre el tiempo en responder a un ítem y el tiempo empleado en responder a cuatro ítems. Más allá de este rango de subitizing, los tiempos de respuestas se incrementan de manera lineal, dependiendo del número de ítems que haya que aprehender, lo que sugiere un proceso de contenido secuencial.

Son numerosos los estudios que han analizado el proceso de subitizing en los niños oyentes con dificultades matemáticas. Koontz y Berch (1996) encontraron que aquellos niños que presentaban dificultades en el aprendizaje de las matemáticas tenían dificultades para realizar la tarea de subitizing y, en su lugar, utilizaban una estrategia de conteo que consumía mucho tiempo. Esta estrategia de conteo era usada incluso en conjuntos inferiores a tres elementos. Landerl, Bevan y Butterworth (2004) encontraron que aquellos niños con dificultades matemáticas que presentaban a la vez dificultades en la lectura presentaban unos tiempos de respuesta que se iban incrementando linealmente. Esta gradación progresiva la mostraban incluso dentro del rango de subitizing. Estos datos sugerían que se estaba produciendo un proceso de conteo más que una aprehensión global dentro del rango de subitizing, que era lo esperado. Sin embargo, las comparaciones con los grupos de control (niños normales y niños con dificultades en lectura) no mostraron efectos significativos.

En el trabajo de Bull et al. (2006) se evaluaron las habilidades de subitizing de niños sordos y de niños oyentes. Cada presentación de estímulos contenía entre uno y seis elementos los cuales eran mostrados en tres formatos diferentes: ordenados de manera aleatoria en el centro de la pantalla con algunos elementos periféricos; como un ordenamiento irregular de puntos (de hecho con dos subgrupos de puntos, lo que presumiblemente requería dos exploraciones oculares) y, finalmente, siguiendo un orden previsible similar al que aparece en los dados, es decir, de manera claramente ordenada. Cada presentación era mostrada durante un periodo de tiempo muy breve (50 milisegundos) para evitar la posibilidad de que los niños contaran los ítems. Debido a que se sabe que los sujetos sordos muestran mejores habilidades en algunos aspectos del procesamiento visual espacial (Rettenback, Diller y Sireteaunu, 1999), se anticipó que las personas sordas deberían realizar bien la tarea de subitizing, 
particularmente en las condiciones en las que los estímulos no eran presentados en una forma canónica bien conocida y donde el proceso de subitizing podía requerir un cambio rápido del foco de atención desde el primer grupo de elementos al segundo o, en el primer caso, una exploración de la zona periférica del conjunto. De hecho, los patrones de resultados fueron muy similares tanto en sordos como en oyentes en las tareas usadas aquí. Si los participantes eran capaces de enumerar rápidamente conjuntos de pequeño tamaño (subitizing) entonces se esperaría una mayor precisión para aquellos conjuntos cuyo tamaño caía dentro de rango de subitizing (1-4) comparado con aquellos conjuntos que presentaran un número de ítems superior al rango de subitizing (5-6). En todos los formatos de presentación, la precisión fue muy alta en la tarea de subitizing (85\%-97\%). La precisión se mantuvo alta para aquellos conjuntos con mayor número de ítems que se ajustaban al formato de presentación ordenado (el de tipo dado), presumiblemente porque todos los participantes eran capaces de usar su conocimiento de los patrones regulares para estimar de manera precisa el número presentado. En el caso de la presentación de los puntos de manera aleatoria o de manera no ordenada, tanto un grupo como otro (sordos y oyentes) mostraron una caída significativa en el rendimiento (hasta aproximadamente el 70\% de precisión). Las personas sordas no mostraron la ventaja que se les había supuesto anteriormente en el caso del formato parcialmente desordenado pero mostraron claramente los efectos típicos de subitizing con un rendimiento similar al del grupo de oyentes. Investigaciones más recientes (Hauser, Dye, Boutla, Green y Bavelier, 2007) apoyan estos resultados. Los sordos hablantes nativos del lenguaje de signos muestran los resultados esperables de la tarea de enumeración: mayor velocidad de conteo y mayor precisión dentro del rango de subitizing e incremento en los tiempos de respuestas y en los errores para aquellas presentaciones que contenían magnitudes fuera del rango de subitizing (entre cinco y diez elementos). Este rendimiento fue completamente comparable con el de sus iguales oyentes en las presentaciones en las que el conjunto de datos o bien estaba en el centro de la pantalla o bien en la periferia de la misma. Hauser et al. (2007) también calcularon el 'punto de ruptura', definido como el número de elementos en el cual se produce el cambio de la estrategia de subitizing a una estrategia de conteo. De nuevo no hubo diferencias significativas en este punto de ruptura, mostrando los dos grupos, sordos y oyentes, un punto de ruptura aproximadamente en el elemento 4 que es el límite habitual del rango de subitizing.

Finalmente, otros resultados en niños sordos (con edades comprendidas entre los 10 y los 13 años) no muestran diferencias en las tareas de subitizing. En este estudio se presentaba a los niños un estímulo durante $200 \mathrm{~ms}$. que contenía entre 2 y 8 ítems. Los participantes tenían que decir cuántos elementos había en la presentación. La precisión en aquellas presentaciones que tenían entre dos 
y cuatro elementos fue muy alta, disminuyendo esta precisión a medida que se iba incrementando el número de elementos simultáneos en la presentación. En general, parece que las ventajas que proporciona la sordera en algunos aspectos de atención visual no conllevan cambios similares en el número de objetos que pueden ser atendidos. Si fuera así, deberíamos esperar que el rango de subitizing se incrementase. Y esto no ocurre. Por otro lado, los resultados muestran que, en contraste con los hallazgos de Koontz y Berch (1996) y de Landerl et al. (2004) con sujetos oyentes, las personas sordas no tienen un déficit en la habilidad de contar conjuntos de objetos, lo que sugiere que la habilidad de subitizing no contribuye a las dificultades matemáticas específicas de los sujetos sordos. Por tanto, hemos de suponer que la fuente de sus dificultades está en otro lado o en otra combinación de variables.

\section{III.5. REPRESENTACIÓN LINEAL O REPRESENTACIÓN LOGARÍTMICA: LA APORTA- CIÓN DEL PROCESO DE ESTIMACIÓN}

Un aspecto básico del sentido numérico es el desarrollo de la estimación numérica. Esta habilidad es necesaria para completar tareas tales como estimar el número de objetos en un conjunto dado cuando están fuera del rango de subitizing (más de cuatro elementos) o para situar las magnitudes numéricas a lo largo de una línea numérica (Barth et al., 2006; Booth y Siegler, 2006). Esta habilidad ha mostrado una fuerte relación positiva con las habilidades de conteo y otras habilidades aritméticas, como puede ser una mejor comprensión de los conceptos matemáticos (LeFevre, Greenham y Waheed, 1993). En general esta habilidad parece covariar con los resultados en los tests de rendimiento matemático desde edades tempranas (preescolares) hasta el tercer grado -nueve años aproximadamente- (Booth y Ziegler, 2006).

Una de las posiciones teóricas acerca de la representación mental numérica sostiene que nuestro sistema interno de representación de magnitudes numéricas se ajusta al sistema de los logaritmos numéricos. Esto quiere decir que vamos posicionando en una línea numérica las magnitudes de forma tal que a medida que las magnitudes numéricas van aumentado, la posición de estas magnitudes sobre la línea se va comprimiendo sin mantener una relación constante entre ellas (Feigenson, Dehaene y Spelke, 2004). Los estudios con adultos y niños y los estudios de habituación de bebés han mostrado que la rapidez y precisión en la comparación de magnitudes numéricas decrece logarítmicamente a medida que la ratio de los números que se comparan se aproxima a uno (efecto de distancia). También han mostrado que esta rapidez y precisión decrecen también a medida que decrecen las magnitudes numéricas cuando comparamos números separados por igual distancia (efectos de tamaño). 
A lo largo del proceso de escolarización, los juicios que los niños realizan sobre la línea numérica progresivamente van adoptando un formato lineal (Siegler y Booth, 2004), en el que las diferencias entre números consecutivos son idénticas independientemente de la posición sobre la línea. Para la comprensión de los conceptos matemáticos, los niños necesitan reconocer la naturaleza lineal de la cantidad y la idea de que todas las partes de un rango son igualmente importantes es clave si se quieren hacer aproximaciones con precisión, si se quieren verificar respuestas aritméticas, etc.

El modelo de representación múltiple de Siegler (1996) sugiere que los sujetos disponen de ambos tipos de representaciones: tanto las representaciones lineales como las logarítmicas, y que ambas se encuentran disponibles de manera intercambiable. La elección de una o de otra dependerá de cuál sea la mejor representación posible para una tarea particular en un contex to concreto. En algunos casos el enfoque logarítmico podrá ser más adecuado que el enfoque lineal (por ejemplo para estimar la cantidad de violinistas necesarios para aumentar el sonido de violín en una orquesta, que sabemos que no guarda una relación lineal), mientras que en otros casos, una representación lineal debería ser más beneficiosa, por ejemplo, allí donde todas las partes de un conjunto son igualmente importantes (por ejemplo en la estructura del sistema para la comprensión de la aritmética en la notación decimal). Se cree que debido a la falta de familiaridad con los números grandes, los niños (y también los adultos en el contexto de números de muy alta magnitud) tienden a basarse en una representación más intuitiva, de tipo logarítmico, para proceder a la estimación. A medida que el niño crece y a medida que se gana en experiencia, esta representación logarítmica es sustituida por una representación lineal que resulta ser la más apropiada.

Un método directo de medida de estas representaciones mentales es examinar la precisión de la estimación numérica en tareas que relacionan un número y una posición en la línea numérica. En una tarea típica de esta naturaleza se presenta una línea con el número cero (0) en un extremo y el número 100, 1000 ó 10000 en el otro dependiendo del contexto. Se proporciona al niño un determinado número y se le pide que estime en qué posición debería ir colocado en la línea numérica. Esa tarea nos proporciona una medida física directa de la representación mental del sujeto acerca de las distancias entre los números en la línea. Usando ese tipo de tareas, Siegler y Booth (2004) observaron un importante cambio evolutivo. Se iba pasando de una representación logarítmica a una representación lineal. El tiempo necesario para realizar esta evolución dependía de la familiaridad con el rango numérico. Por ejemplo, los niños de preescolar (con edades comprendidas entre los 5 y los 6 años) que tenían una experiencia muy limitada con números entre el 1 y el 100, mostraron un patrón de representación numérica claramente logarítmico. En cambio, aquellos niños 
de edades un poco superiores (entre los 6 y los 7 años) ya obtuvieron mejores resultados, con la mitad del grupo de edad mostrando patrones logarítmicos y la otra mitad ajustándose a un patrón lineal. Los niños del curso siguiente (con edades comprendidas entre los 7 y los 8 años) que ya tenían una mayor familiaridad con los números de ese rango era más probable que mostraran representaciones numéricas que se ajustaran una función lineal. En el contexto de una línea de 0 a 1000, este cambio aparece alrededor del cuarto grado educativo (alrededor de 10 años), reforzando la idea de que el cambio de una representación logarítmica a una representación lineal es facilitada por la mayor experiencia y familiaridad con el rango numérico (Siegler y Opfer, 2003). Esta experiencia y familiaridad se obtiene a partir de las oportunidades que el niño tenga para el aprendizaje incidental mediante, por ejemplo, juegos que proporcionen una combinación de pistas espaciales, temporales, cinestésicas y auditivo-verbales. Estas pistas serán las que se usen para el cálculo de las magnitudes numéricas (Whyte y Bull, 2008).

Si los niños sordos tuvieran problemas para hacer esa transición desde la representación logarítmica natural al sistema lineal enseñado por la escuela, entonces también deberían mostrar evidencias de una mayor frecuencia de representaciones logarítmicas. En el caso de los adultos sordos debería darse que si la exposición a números de valores altos (hasta 10.000) ocurre con menos frecuencia o si presentan dificultades para inhibir la representación logarítmica natural de los números (Geary et al., 2007b), entonces deberíamos esperar de ellos menores representaciones lineales de magnitud o menor precisión a la hora de colocar los números sobre una escala lineal.

\section{III.6. LA ESTIMACIÓN NO SIMBÓLICA DE MAGNITUDES}

La habilidad para realizar estimaciones también ha sido estudiada mediante el uso de tareas no simbólicas. Los estudios que han valorado las habilidades y dificultades matemáticas de los niños han argumentado que el problema puede radicar en el acceso a la magnitud numérica a partir de símbolos más que en el propio procesamiento numérico en sí mismo. Rouselle y Noel (2007) encontraron que los niños que presentaban dificultades matemáticas únicamente eran deficitarios cuando comparaban dígitos arábigos (comparaciones numéricas simbólicas) pero no lo eran cuando comparaban colecciones de ítems (magnitudes numéricas no simbólicas). Las habilidades numéricas observadas en estudios con animales, con bebés y con adultos sugería la existencia de una capacidad primitiva preverbal de estimación de la magnitud que está presente con anterioridad a la emergencia de las representaciones numéricas simbólicas. Esta capacidad produciría un patrón característico de resultados que mostraría que cuando la ratio entre las dos cantidades que se están comparando está cer- 
cana al 1, entonces la precisión del juicio de estimación disminuye claramente (Barth et al., 2006). En estos estudios, a los participantes se les pide que realicen aproximaciones, discriminaciones, o incluso cálculos aritméticos, a partir de presentaciones, durante un tiempo de exposición muy breve, de conjuntos de objetos donde no es posible el conteo utilizando los medios simbólicos tradicionales. Los estudios con neuroimagen han puesto de manifiesto la importancia de estas representaciones no simbólicas pues se ha mostrado que, incluso en aquellas tareas que tienen que ver con el cálculo simbólico exacto, también se activan automáticamente las representaciones numéricas no simbólicas aunque no sean necesarias para la tarea (Dehaene et al., 1990).

Masataka (2006) comparó adultos sordos y adultos oyentes en tareas como las que fueron propuestas por Barth et al. (2006). En una tarea de comparación, a los sujetos se les mostraban dos conjuntos de elementos que variaban en una proporción de $0,75,0,80,0,83$ y 0,86 y se les pidió que marcaran cuál de los dos conjuntos contenía más elementos. Los sujetos completaron también una tarea de sustracción no simbólica, en la que se les pidió que restasen un segundo conjunto a partir de un primero. Luego, que compararan éste con el número de elementos de un tercer conjunto para, finalmente, decidir si el tercer conjunto contenía más o menos elementos que la resta realizada anteriormente. Los participantes también realizaron una tarea formal de matemáticas en la que se utilizó el conjunto de estímulos utilizados para la tarea de sustracción no simbólica sólo que sustituyendo los elementos no simbólicos por símbolos numerales arábigos. En general, los resultados en precisión en la tarea de comparación no mostraron diferencias significativas entre los sujetos sordos y los sujetos oyentes. Sin embargo los sujetos sordos fueron significativamente más precisos en la tarea de sustracción no simbólica que en la simbólica. Ambos grupos, sordos y oyentes, mostraron el típico efecto de la ratio, mostrando una precisión que iba declinando a medida que la diferencia de ratio entre los dos subconjuntos se aproximaba a 1 (uno).

Tomados en su conjunto, estos resultados sugieren que la disminución en precisión en las tareas de estimación, tanto para los sordos como para los oyentes con dificultades matemáticas, puede aparecer únicamente cuando hay que acceder al significado de los números a partir de los símbolos (este efecto fue formulado con el nombre de "hipótesis del déficit de acceso" por Rousselle y Noël, 2007). Cuando la estimación se basa en elementos no simbólicos, la precisión se incrementa. Estas autoras han recomendado realizar más investigaciones acerca de las habilidades de estimación, incluyendo tareas que impliquen tanto componentes simbólicos (por ejemplo respuestas a problemas aritméticos, problemas con cantidades de dinero) y de estimación no simbólica (por ejemplo, estimación de la distancia entre dos puntos, estimar el número de elementos de un conjunto). Esa cuestión parece particularmente importante si 
tenemos en cuenta otros hallazgos que muestran que unas buenas habilidades de estimación están relacionadas positivamente con los resultados matemáticos posteriores (Booth y Siegler, 2006; Siegler y Booth, 2004). La habilidad para realizar estimaciones precisas puede basarse en parte en la habilidad para controlar las propias respuestas, en la habilidad para considerar la adecuación de una estimación y, en consecuencia, para ajustarse a ella. Una habilidad que puede que no sea usada con el mismo nivel de eficiencia por los niños sordos y por oyentes.

\section{CONCLUSIÓN}

El procesamiento numérico de las personas sordas ha de ser estudiado en el contexto de un funcionamiento cognitivo general mediado por el hecho de la sordera. Este hecho, el ser sordo, no se limita a la mera cuestión de carecer de audición sino que produce un considerable impacto sobre muchas de las habilidades necesarias para un eficiente funcionamiento cognitivo. Así, es posible contemplar un funcionamiento deficitario en muchas áreas de funcionamiento cognitivo sin que las escasas habilidades hiperdesarrolladas en las personas sordas, como el caso de la atención y percepción visual se traduzcan, a la hora de la verdad, en ventajas evidentes en otras áreas de funcionamiento cognitivo, tal como sostenían en su día los defensores de las estrategias compensatorias. El caso del procesamiento numérico muestra una evidencia de mal funcionamiento del sistema cognitivo. Afortunadamente este mal funcionamiento, aunque evidente, no presenta unos niveles tan bajos, comparados con los sujetos oyentes, como los observados en el caso de la lectura. Los estudios han puesto de manifiesto que este desfase existe y que se mantiene a pesar de todo el proceso de escolarización y de instrucción formal en matemáticas. Pero, y es un argumento a favor de las personas sordas, existe un buen porcentaje de niños sordos que manifiestan habilidades similares a la media de los niños oyentes e incluso mejores. Ello permite suponer que las dificultades en procesamiento numérico no son tan estructurales ni tan difíciles de resolver como las que se manifiestan en el caso de la lectura que citábamos antes. Presentan en ocasiones un funcionamiento similar al de los niños oyentes que presentan dificultades de aprendizaje matemático, lo que permite suponer que están implicados los mismos mecanismos. Esto es particularmente interesante pues suele coincidir con aquellos niños con dificultades matemáticas que presentan a la vez un cierto trastorno en lectura. Ello nos habla de un componente compartido que tiene que ver con la naturaleza simbólica de las representaciones numéricas. A la luz de las investigaciones que hemos presentado podemos sospechar que se trata más bien de un funcionamiento empobrecido, debido al impacto de muchas otras 
variables cognitivas que funcionan deficitariamente en las personas sordas, que de una verdadera carencia estructural. Los mecanismos básicos de comparación de magnitudes, de enumeración, de conteo, de aprehensión súbita (subitizing), aunque empobrecidos, se hallan presentes. Y ello es esperanzador para el futuro educativo y social de las personas sordas. Las futuras investigaciones nos permitirán afianzarnos, o no, en esta conclusión.

\section{REFERENCIAS}

ANSARI, D., y KARMILOFF-SMITH, A. (2002). "Atypical trajectories of number development: A neuroconstructivist perspective". Trends in Cognitive Sciences, 6, 511-516.

AUSTIN, G. F. (1975). "Knowledge of selected concepts obtained by an adolescent deaf population". American Annals of the Deaf, 120, 360-370.

BANDURSKI, M., y GALKOWSKI, T. (2004). "The development of analogical reasoning in deaf children and their parents' communication mode". Journal of Deaf Studies and Deaf Education, 9, 153-175.

BARTH, H., LA MONT, K., LIPTON, J., DEHAENE, S., KANWISHER, N. y SPELKE, E. (2006). "Non-symbolic arithmetic in adults and young children". Cognition, 98, 199-222.

BERCH, D. B. (2005). "Making sense of number sense: Implications for children with mathematical disabilities". Journal of Learning Disabilities, 38, 333-339.

BOOTH, J. L., y SIEGLER, R. S. (2006). "Developmental and individual differences in pure numerical estimation". Developmental Psychology, 41, 189-201.

BULL, R., BLATTO-VALLEE, C., y FABICH, M. (2006). "Subitizing, magnitude representation, and magnitude retrieval in deaf and hearing adults". Journal of Deaf Studies and Deaf Education, 11, 289-302.

BULL, R., MARSCHARK, M., y BLATTO-VALLEE, G. (2005). "SNARC hunting: Examining number representation in deaf students". Learning and Individual Differences, 15, 223-236.

BUTTERWORTH, B. (2005). "The development of arithmetical abilities". Journal of Child Psychology and Psychiatry, 46, 3-18.

DEHAENE, S. (1992). "Varieties of numerical abilities". Cognition, 44, 1-42.

DEHAENE, S. (1997). The number sense: How the mind creates mathematics. New York: Oxford University Press.

DEHAENE, S., BOSSINI, S., y GIRAUX, P. (1993). "The mental representation of parity and number magnitude". Journal of Experimental Psychology: General, 122, 371-396. 
DEHAENE, S., y CHANGEUX, J. (1993). "Development of elementary numerical abilities: A neuronal model". Journal of Cognitive Neuroscience, 5, 390-407.

DEHAENE, S., DEHAENE-LAMBERTZ, G., y COHEN, L. (1998). “Abstract representation of number in the animal and human brain". Nature Neuroscience, 21, 355-361.

DEHAENE, S., DUPOUX, E., y MEHLER, J. (1990). "Is numerical comparison digital: Analogical and symbolic distance effects in two-digit number comparison". Journal of Experimental Psychology: Human Perception and Performance, 16, 626-641.

FROSTAD, P., y AHLBERG, A. (1999). "Solving story-based arithmetic problems: Achievement of children with hearing impairment and their interpretation of meaning". Journal of Deaf Studies and Deaf Education, 4(4), 283-293.

GALLISTEL, C. R., y GELMAN, R. (1992). "Preverbal and verbal counting and computation". Cognition, 44, 43-74.

GIRELLI, L., LUCANGELI, D., y BUTTERWORTH, B. (2000). "The developmental of automaticity in accessing number magnitude". Journal of Experimental Child Psychology, 76, 104-122.

GREGORY, S. (1998). "Mathematics and deaf children". In S. Gregory, P. Knight, W. McCracken, S. Powers, y L. Watson (Eds.), Issues in Deaf Education (pp. 119-128). London: Fulton.

HAUSER, P. C., DYE, M.W.G., BOUTLA, M., GREEN, C. S., y BAVELIER, D. (2007). "Deafness and visual enumeration: Not all aspects of attention are modified by deafness". Brain Research, 1153, 178-187.

HYDE, M., ZEVENBERGEN, R., y POWER, D. (2003). "Deaf and hard of hearing students' performance on arithmetic word problems". American Annals of the Deaf, 148(1), 56-64.

JORDAN, N. C., HANICH, L. B., y KAPLAN, D. (2003). “A longitudinal study of mathematical competencies in children with specific math difficulties versus children with comorbid mathematics and reading difficulties". Child Development, 74, 834-850.

KELLY, R. R., LANG, H. G., y PAGLIARO, C. M. (2003). "Mathematics word problem solving for deaf students: A survey of practices in Grades 6-12". Journal of Deaf Studies and Deaf Education, 8, 104-119.

KOONTZ, K. L. y BERCH, D. B. (1996). "Identifying simple numerical stimuli: Processing inefficiencies exhibited by arithmetic learning disabled children". Mathematical Cognition, 2, 1-23.

LANDERL, K., BEVAN, A., y BUTTERWORTH, B. (2004). "Developmental dyscalculia and basic numerical capacities: A study of 8-9 year old students". Cognition, 93, 99-125. 
LANG, H. G. (2003). "Perspectives on the history of deaf education". In M. Marschark y P. E. Spencer (Eds.), Oxford handbook of deaf studies, language, and education (pp. 9-20). New York: Oxford University Press.

LEFEVRE, J. A., GREENHAM, S. L., y WAHEED, N. (1993). "The development of procedural and conceptual knowledge in computational estimation". Cognition and Instruction, 11, 95-132

MacLEOD, C. M. (1991). "Half a century of research on the Stroop effect: An integrative review". Psychological Bulletin, 109, 163-203.

MARSCHARK, M., LANG, H., y ALBERTINI, J. (2002). Educating deaf students: From research to practice. New York: Oxford University Press.

MASATAKA, N. (2006). "Differences in arithmetical subtraction of nonsymbolic numerosities by deaf and hearing adults". Journal of Deaf Studies and Deaf Education, 11, 139-143.

MAZZOCCO, M. M. M. (2005). "Challenges in identifying target skills for math disability screening and intervention". Journal of Learning Disabilities, 38, 318-323.

McCLOSKEY, M. (2007). "Quantitative literacy and developmental dyscalculias". In D. B. Berch y M.M.M. Mazzocco (Eds.), Why is math so hard for some children? The nature and origins of mathematical learning difficulties and disabilities (pp. 415-429). Baltimore: Paul H. Brookes.

PETERSON, S., y SIMON, T. J. (2000). "Computational evidence for the subitizing phenomenon as an emergent property of the human cognitive architecture". Cognitive Science, 24, 93-122.

PIAZZA, M., y DEHAENE, S. (2005). "From number neurons to mental arithmetic: The cognitive neuroscience of number sense". In M. S. Gazzaniga (Ed.), The cognitive neourosciences (3rd ed., pp. 865-875). Cambridge, MA: MIT Press.

POWERS P, GREGORY S, y THOUTENHOOFD E. (1998). The Educational Achievements of Deaf Children. DfEE Research Report 65. London: Crown Copyright.

RETTENBACK, R., DILLER, C., y SIRETEAUNU, R. (1999). "Do deaf people see better? Texture segmentation and visual search compensate in adult but not in juvenile subjects”. Journal of Cognitive Neuroscience, $11,560-583$.

ROUSSELLE, L., y NOËL, M-P. (2007). "Basic numerical skills in children with mathematical learning disabilities: A comparison of symbolic vs. nonsymbolic number magnitude processing". Cognition, 102, 361-395.

RUBINSTEN, O. y HENIK, A. (2005). "Automatic activation of internal magnitudes: A study of developmental dyscalculia”. Neuropsychology, 19,641-648. 
RUBINSTEN, O., HENIK, A., BERGER, A., y SHAHAR-SHALEV, S. (2002). "The development of internal representations of magnitude and their association with Arabic numerals". Journal of Experimental Child Psychology, 81, 74-92.

SCHICK, B. (2005). "Educational interpreting and cognitive development in children: Potential relationships". In E. Winston (Ed.), Educational interpreting: The questions we should be asking (pp. 73-87). Washington, DC: Gallaudet University Press.

SHALEV, R. S. (2007). "Prevalence of developmental dyscalculia. In D.B. Berch and M.M.M. Mazzocco (Eds.), Why is math so hard for some children? The nature and origins of mathematical learning difficulties and disabilities (pp. 49-63). Baltimore: Paul H. Brookes.

SIEGLER, R. S. (1996). Emerging minds: The process of change in children's thinking. New York: Oxford University Press.

SIEGLER, R. S., y BOOTH, J. (2004). "Development of numerical estimation in young children". Child Development, 75, 428-444.

SIEGLER, R. S., y OPFER, J. E. (2003). "The development of numerical estimation: Evidence for multiple representations of numerical quantity". Psychological Science, 14, 237-243.

SWANWICK, R., ODDY, A., y ROPER, T. (2005). "Mathematics and deaf children: An exploration of barriers to success". Deafness and Education International, 7, 1-21.

TRAXLER, C. B. (2000). "Measuring up to performance standards in reading and mathematics: Achievement of selected deaf and hard-of-hearing students in the national norming of the 9th Edition Stanford Achievement Test". Journal of Deaf Studies and Deaf Education, 5, 33 7-348.

WHYTE, J. C., y BULL, R. (2008). "Number games, magnitude representation, and basic number skills in preschoolers". Developmental Psychology, 44,588-596.

WOOD, H. A.,WOOD, D. J., KINGSMILL, M. C., FRENCH, J. R. W. y HOWARTH, P. (1984). "The mathematical achievement of deaf children from different educational environments". British Journal of Educational Psychology 54, 254-264.

XU, F., y SPELKE, E. (2000). "Large number discrimination in 6-month-old infants". Cognition, 74, B1-B11. 